\title{
Interpretation bias and anxiety in childhood: stability, specificity and longitudinal associations
}

Article

Published Version

Creswell, C. and O'Connor, T. G. (2011) Interpretation bias and anxiety in childhood: stability, specificity and longitudinal associations. Behavioural and Cognitive Psychotherapy, 39 (02). pp. 191-204. ISSN 1469-1833 doi:

https://doi.org/10.1017/S1352465810000494 Available at https://centaur.reading.ac.uk/22031/

It is advisable to refer to the publisher's version if you intend to cite from the work. See Guidance on citing.

Published version at: http://dx.doi.org/10.1017/S1352465810000494

To link to this article DOI: http://dx.doi.org/10.1017/S1352465810000494

Publisher: Cambridge University Press

All outputs in CentAUR are protected by Intellectual Property Rights law, including copyright law. Copyright and IPR is retained by the creators or other copyright holders. Terms and conditions for use of this material are defined in the End User Agreement.

www.reading.ac.uk/centaur 
Central Archive at the University of Reading

Reading's research outputs online 


\title{
Interpretation Bias and Anxiety in Childhood: Stability, Specificity and Longitudinal Associations
}

\author{
Cathy Creswell \\ University of Reading, $U K$ \\ Thomas G. O’Connor \\ University of Rochester Medical Center, USA
}

\begin{abstract}
Background: Biases in the interpretation of ambiguous material are central to cognitive models of anxiety; however, understanding of the association between interpretation and anxiety in childhood is limited. To address this, a prospective investigation of the stability and specificity of anxious cognitions and anxiety and the relationship between these factors was conducted. Method: Sixty-five children (10-11 years) from a community sample completed measures of self-reported anxiety, depression, and conduct problems, and responded to ambiguous stories at three time points over one-year. Results: Individual differences in biases in interpretation of ambiguity (specifically "anticipated distress" and "threat interpretation") were stable over time. Furthermore, anticipated distress and threat interpretation were specifically associated with anxiety symptoms. Distress anticipation predicted change in anxiety symptoms over time. In contrast, anxiety scores predicted change in threat interpretation over time. Conclusions: The results suggest that different cognitive constructs may show different longitudinal links with anxiety. These preliminary findings extend research and theory on anxious cognitions and their link with anxiety in children, and suggest that these cognitive processes may be valuable targets for assessment and intervention.
\end{abstract}

Keywords: Cognition, anxiety, children, interpretation biases.

\section{Introduction}

Processing biases are central to the cognitive model of anxiety (e.g. Beck, Emery and Greenberg, 1985). Specifically, a tendency to interpret increased threat and anticipate reduced coping are thought to reinforce avoidant behaviour and anxious affect in a circular, selfreinforcing manner. Based on this model, strategies to target these interpretation biases shape treatments of anxious adults and children as young as 7 years of age (e.g. Barrett, Dadds and Rapee, 1996; Kendall, 1994). However, although these biases are linked with anxiety in children (e.g. Barrett, Rapee, Dadds and Ryan, 1996), evidence is lacking in relation to the

\footnotetext{
Reprint requests to Cathy Creswell, Clinical Research Fellow, Winnicott Research Unit, School of Psychology, University of Reading, 3 Earley Gate, Whiteknights, PO Box 218, Reading RG6 6AL, UK. E-mail: c.creswell@ reading.ac.uk
} 
degree of anxiety-specificity of associations, the temporal stability of "anxious cognitions" and their associations with anxiety over time during childhood. This preliminary prospective investigation examines the stability and anxiety-specificity of anxiety-related cognitive biases, interpretation bias towards threat and anticipation of distress, and their longitudinal links with anxious affect in late childhood.

Various paradigms have been used to assess interpretation biases in youth, including interpretation of homophones, ambiguous facial expressions and, most commonly, ambiguous stories (see e.g. Hadwin, Garner and Perez-Olivas, 2006). Within the latter paradigm, individuals are presented with a series of scenarios in which it is not quite clear what is happening and an interpretation is necessary. The finding that anxious children show (i) an increased tendency to interpret threat and (ii) anticipate increased negative emotion compared with their non-anxious peers has been widely replicated (Barrett et al., 1996; Bögels, and Zigterman, 2000; Chorpita, Albano and Barlow, 1996; Muris, Luermans, Merckelbach and Mayer, 2000; Muris, Merckelbach and Damsma, 2000; Muris, Rapee, Meesters, Shouten and Geers, 2003). Whether or not these findings are specific to anxiety, however, remains unclear. Indeed, few studies have included assessment of other psychosocial difficulties, and where clinical comparison groups have been included, associations have not always been specific to highly anxious groups. For example, Barrett et al. (1996) reported similar threat interpretation biases amongst clinically anxious and oppositional youth, and Eley et al. (2008) found that associations with negative interpretations were independently associated with symptoms of depression but not anxiety. The current study adds to research conducted to date by examining the anxiety-specific nature of both threat interpretation and anticipated distress, and including assessments of anxiety, depression and behavioural problems. The inclusion of anticipated distress is based on previous findings that anxiety is not only associated with threat interpretation biases but also a tendency to anticipate negative emotional reactions in response to ambiguity in comparison to non-anxious peers (e.g. Bögels and Zigterman, 2000).

Another important limitation of existing studies is the reliance on cross-sectional data; we know little about the natural course of anxious cognitions in children and their association with anxiety symptoms over time. Given the limited prior work, our developmental hypotheses are guided by research in depressed and aggressive cognitions. One consistent finding from the depression literature is that the association between cognitive style and symptoms of depression may become more robust from middle childhood to mid-adolescence (e.g. NolenHoeksema, Girgus and Seligman, 1986; Nolen-Hoeksema, Girgus and Seligman, 1992; Turner and Cole, 1994). For example, Nolen-Hoeksema et al. (1992) found that life events but not cognitive style (in this case pessimistic explanatory style) predicted depressed affect in 6-year-olds, whereas cognitive style did predict change in depressed affect in adolescence. Furthermore, cognitions associated with aggression have been found to be more strongly associated with aggressive behaviour in adolescence than in pre-adolescence, and behaviour seemed to precede changes in beliefs until adolescence, when beliefs began to precede changes in behaviour (Huesmann and Guerra, 1997; Lansford et al., 2006). The implication is that cognitive style may manifest as, and act as a risk for, psychopathology once cognitive styles become more stable with increasing cognitive maturity and/or experience.

Whether or not this developmental hypothesis extends to anxiety is unclear. For example, cognitive biases in anxiety may represent a threat-detection mechanism that is an integral part of the emotion of anxiety and not subject to comparable change (Taghavi, Neshat-Doost, Moradi, Yule and Dalgleish, 1999) (i.e. cognitive bias would be strongly associated with 
anxiety across childhood because it is part of the phenomenology rather than a separate risk for anxiety).

What is clear is that prospective investigations are needed that consider stability and change in cognitive bias and affect without confounding age effects, i.e. by restricting samples to narrow age ranges, and allowing for bi-directional associations. In relation to anxious cognitions, we are aware of one study that has addressed some of these aims. Muris, Jacques and Mayer (2004) conducted a 4-week prospective investigation, with children aged 9-13 years, of the association between anxiety symptoms and indices of threat perception. Their results suggested that anxious cognitions were moderately stable over time; however there was no evidence for a direct prospective association between threat perception and change in anxiety symptoms over time. The authors questioned whether 4 weeks was a sufficient timeperiod of assessment, and suggested a replication of the study over a longer time period to increase the likelihood of clear changes in anxiety symptoms between assessments, and hence greater potential to detect predictors of change. That is addressed in the current longitudinal study, which followed children for a 1-year period and capitalized on a normative stress - the transition to secondary school - to test hypotheses about the change over time in anxious cognitions and emotion. This transition marks a time of change in school environment, academic and social activities (Robinson, Garber and Hilsman, 1995) and has been associated with increased psychological distress and problem behaviour (e.g. Robinson et al., 1995; Chung, Elias and Schneider, 1998); it may therefore provide a natural experiment for assessing intra-individual differences and exacerbation of anxious cognitions and affect.

In summary, we conducted a prospective longitudinal study to address the following questions:

1) Are threat interpretation and distress anticipation specifically associated with anxiety symptoms, in comparison to other indices of emotional and behavioural adjustment (depression and oppositional behaviour)?

2) Are interpretation biases towards high threat and anticipation of high distress when confronted with ambiguity stable over a 1-year period?

3) Does cognitive style, assessed prior to a naturalistic stressor, predict change in anxiety symptoms in children after exposure to a major life event; is there evidence of a bi-directional association?

\section{Method}

\section{Sample}

The participants were 65 children ( 30 boys and 35 girls) from UK year 6 (aged 10-11 years) recruited from 14 mixed sex, state primary schools in North London. Full details of the sample are provided elsewhere (Creswell, O'Connor and Brewin, 2006 ${ }^{1}$ ). In brief, the sample represented $27 \%$ of the invited children; despite the low take-up rate the obtained sample was representative of the invited group on gender composition, frequency of special-educational

\footnotetext{
${ }^{1}$ This formed part of a study addressing this question, and parental influences on the development of anxious cognitions reported elsewhere (Creswell, O'Connor and Brewin, 2006) as such parental participation was also required for children to be recruited to the study, which had a negative impact on participation rates.
} 
needs, and single-parent family status, but were more likely to be White-British (56\% versus $\left.40 \% ; \chi^{2}(2)=6.18, p<.05\right)$ and less likely to be receiving free school meals $(25 \%$ versus $\left.46 \% ; \chi^{2}(1)=8.03, p<.005\right)$, response biases commonly observed in developmental research.

Eleven of the children were not able to participate in the first follow-up phase of the study (1 parent was deceased, 2 families were untraceable, 4 children declined, 4 parents declined), leaving $54(83 \%)$ children available at the time 2 assessment. For the third assessment, 49 (75\%) families provided data. Participants and non-participants did not differ on any measures at time 2. Compared with those who provided data at all time points, non-respondents at the third phase did not differ on measures of anxiety but were more likely to have special educational needs (29\% non-responders vs. $8 \%$ responders; $\left.\chi^{2}(1)=5.39, p=0.02\right)$ and higher levels of conduct problems on the SDQ at time $1, t(63)=2.46, p=.02$.

\section{Procedure}

For the first two assessments, families who agreed to take part in the study were visited in their home or school on two occasions by doctoral or masters level researchers with a background in psychology and who had been trained on the administration of all measures. After being given both written and verbal information about the study, mothers and children signed informed consent forms. A researcher was present throughout completion of questionnaires in order to introduce procedures, check understanding, and respond to queries. The third assessment was carried out by post unless the families requested assistance completing the measures at home (two families). Each family received payment at each assessment ( $\$ 50$ in total). The study was approved by the University College London and University College London Hospital Research Ethics Committee. The first assessment took place in the final half of the children's final year at primary school, the second assessment took place in the final half of the first term at secondary school ( 5 months later) and the third in the final term of the first year at secondary school (6 months later). The measures below were administered at all time points.

\section{Measures}

Ambiguous Situations Questionnaire (ASQ). Ambiguous scenarios questionnaires are frequently used to assess interpretation of ambiguity and significant associations have consistently been found with anxiety (see e.g. Hadwin et al., 2006). Following Barrett et al. (1996) each child was presented with 12 ambiguous situations (6 physical and 6 social) that could be interpreted as either threatening or non-threatening; situations were presented in random order. Children were then asked to provide a free response to the question: "What do you think is most likely to have happened?" Responses to this open-ended question were scored by a psychology graduate who was blind to the child's scores on other measures. Free responses regarding the cause of the event were coded as "Threat" (e.g. "the dog is going to bite me") vs. "Non-Threat" (e.g. "the dog wants to play") explanations. The number of Threat responses was summed across the 12 situations (range 0-12). "Don't know" responses from the child were scored as "Non-Threat". A second independent coder (psychology graduate) coded a sample of the responses $(n=48)$ in order to assess inter-rater reliability. Intra class correlation, assessed using Shrout and Fleiss' third model for fixed raters (Shrout and Fleiss, 1979), was acceptable, ICC $=.93,95 \%$ CI.88-.96, $F(47)=27.59, p<.001$. The child was 
then presented with a possible threatening and non-threatening explanation and was asked to indicate which of two possibilities was more likely. Order of Threat and Non-Threat items was counter-balanced across the 12 scenarios. The number of Threat choices was totalled across the 12 situations. Responses from the free response and forced choice format Threat questions correlated highly (time $1: r(65)=.80, p<.001$; time $2: r(54)=.80, p<.001$ ) so were summed to create a total score for Threat.

In addition, the ambiguous scenarios questionnaire was modified to include a measure of anticipated distress. For each situation, the child was asked to report on his/her anticipated level of Distress (from 0-10) in that situation. A total score of Anticipated Distress was based on the average score across the 12 situations (range 0-120). Internal reliability of the scale was good (time $1 \alpha=.82$; time $2 \alpha=.84$; time $3 \alpha=.92$ ).

Spence Children's Anxiety Scale (SCAS; Spence, 1998). The SCAS is a widely used self-report questionnaire measuring symptoms of DSM-defined anxiety disorders in children and adolescents. The scale contains 38 anxiety symptom items, as well as positive "filler" items and an open response. Each item is measured on a 4-point scale from "never" (0) to "always" (3). A total score is derived by summing all anxiety symptom scores. Scores are also calculated for six subscales: generalized anxiety (e.g. "I worry that something bad will happen"), separation anxiety ("I feel scared if I have to sleep on my own"), social phobia ("I feel afraid that I will make a fool of myself in front of people"), panic and agoraphobia ("I feel afraid of being in crowded places"), obsessive-compulsive ("I have to think of special thoughts to stop bad things from happening"), and physical injury fears ("I am scared of insects or spiders"). Spence (1997) reports robust internal properties of the scale. Internal consistency was good (time $1 \alpha=.89$; time $2 \alpha=.84$; time $3 \alpha=.93$ ).

Child Depression Inventory (Long form) (CDI; Kovacs, 1979). The CDI is a widely used self-report measure of depressive symptoms for children, with extensive support for its reliability and validity (Kazdin, 1981; Saylor, Finch, Spirito and Bennett, 1984). The scale comprises 27 items, in which the child selects one of three statements representing from a low frequency of the particular depressive symptom (0) to a high frequency of the symptom (2). Internal consistency was good (time $1 \alpha=.80$; time $2 \alpha=.88$; time $3 \alpha=.87$ ).

Strengths and Difficulties Questionnaire-self-report (SDQ-S; Goodman, 1997). The SDQ$\mathrm{S}$ is a child-self-report behavioural screening measure comprising five scales: emotional symptoms, conduct problems, hyperactivity-inattention, peer problems, and pro-social, which have been confirmed by factor analysis (Goodman, 2001). Satisfactory internal consistency, cross-informant correlation and test-retest stability have been reported for children aged 1116 years (Goodman, 2001) and the SDQ is widely used within clinical research. Each subscale is made up of five items, scored on a 3-point scale between "not true" $(0)$ and "certainly true" (2). The current study utilizes the "conduct problems" scale as a measure of oppositional behaviour. Internal consistency was acceptable (time $1 \alpha=.60$; time $2 \alpha=.60$; time 3 $\alpha=.78)$.

\section{Results}

Correlations between ASQ Threat Interpretation and Anticipated Distress scores were generally modest (time $1, r(65)=.27, p=.03$; time $2, r(54)=.20, p=.14$; time $3, r(49)=.55$, 
$p<.001)$ and were therefore considered as separate constructs. Data were screened in accordance with Tabachnick and Fidell's (1996) recommendations for screening univariate ungrouped data. One outlying data point was identified on child self-report on the CDI at time 3, and hence was excluded from analyses involving this variable. Scores on the SDQ were positively skewed at all time points, as was CDI at the first and second time point and ASQ Threat at the third time point. Analyses involving these variables was replicated using logarithmic or square root transformations as appropriate; all results were consistent with those using untransformed data so are not given here (contact the first author for more details). Associations between the key variables and demographic characteristics (gender, ethnicity, eligibility for free school meals and registered special educational needs) were examined. Non-White British children reported significantly greater symptoms and anxiety-related cognitive biases at all three time points(Anxiety symptoms, time $1: t(63)=2.30, p<.05$; time 2: $t(52)=3.44, p<.01$; time $3: t(47)=2.88, p<.01)$; ASQ Anticipated Distress: time $1: t(63)=2.44, p<.05$; time $2: t(52)=2.18, p<.05$; time $3: t(47)=2.46, p<.05$; Threat Interpretation scores (time 1: $t(65)=2.15, p<.05$; time $2: t(52)=3.23, p<.01$; time 3 : $t(49)=2.63, p<.05)$. Non-White British children also reported higher levels of conduct problems at time 1 and 2 (time $1: t(63)=2.46, p<.05$; time $2: t(52)=2.92, p<.01$ ). Other demographic variables did not have such consistent associations; however, at times 1 and 3 girls reported more anxiety symptoms (SCAS; time 1: $t(63)=1.97, p<.05$; time $3: t(47)=$ $2.97, p<.05)$, ASQ anticipated distress (time $1: t(63)=2.46, p<.05$; time $3: 3.42, p<.005$ ) and ASQ threat interpretation at time $3(t(47)=2.10, p<.05)$ than boys. Accordingly, gender and ethnicity were controlled for and interaction effects were explored in later analyses.

\section{Specificity of the association between threat interpretation and distress to anxiety symptoms}

Bivariate correlations between measures of cognition and affect at all time points are presented in Table 1. Robust associations between Anticipated Distress from the ambiguous situations questionnaire and anxiety symptoms (SCAS) were found, and remained statistically significant after controlling for the number of tests (Bonferroni). It can be seen from both cross-sectional and longitudinal analyses that the overlap between Anticipated Distress and anxiety was consistently large, whereas the link with depression and conduct problems was typically small, and non-significant after a Bonferroni correction. In contrast, associations with Threat Interpretation appeared to be moderately associated with both anxiety and depression; however this pattern was less consistent (Table 1), and after Bonferroni correction, Threat Interpretation was only significantly associated with anxiety at time 2 and time 3 .

\section{Stability over time of interpretation bias towards threat and anticipation of distress.}

Two types of change are evaluated to investigate the stability of cognitions and affect: mean level change and change in rank order of individuals over time. As before, Bonferroni corrections for statistical significance were applied to control for the number of tests conducted. Table 2 displays the mean scores on the anxious affect and anxious cognition measures. Repeated measures analyses, based on those participants where data were available at all time points, indicated a significant mean change only for threat interpretation, $F(2,41)=$ $8.01, p=.001$, Effect size of change, $\gamma=.40$, representing a reduction in threat interpretation between the pre (time 1) and post-transition (time 2) assessments. Bivariate correlations 
Table 1. Bivariate correlations between measures of cognitions and affect

\begin{tabular}{|c|c|c|c|c|c|c|}
\hline & \multicolumn{2}{|c|}{ Time 1} & \multicolumn{2}{|c|}{ Time 2} & \multicolumn{2}{|c|}{ Time 3} \\
\hline & ASQ Dis & ASQ Th & ASQ Dis & ASQ Th & ASQ Dis & ASQ Th \\
\hline \multicolumn{7}{|l|}{ Time 1} \\
\hline SCAS & $.53^{* * *}+$ & .23 & $.34^{*}$ & .19 & $.48^{* * *}+$ & $.56^{* * *}+$ \\
\hline CDI & $.27^{*}$ & $.40^{* *}$ & .11 & $.49^{* *}$ & .14 & $.52^{* *}$ \\
\hline SDQ Con & -.19 & $.31^{*}$ & -.21 & $.27^{*}$ & $-.37^{* *}$ & .04 \\
\hline \multicolumn{7}{|l|}{ Time 2} \\
\hline SCAS & $.54^{* * *}+$ & $.30^{*}$ & $.56^{* * *}+$ & $.46^{* * *}+$ & $.52^{* * *}+$ & $.56^{* * *}+$ \\
\hline CDI & .07 & .26 & .06 & $.38^{* *}$ & .17 & $.46^{* *}$ \\
\hline SDQ Con & -.06 & $.36^{*}$ & -.09 & .20 & -.21 & .19 \\
\hline \multicolumn{7}{|l|}{ Time 3} \\
\hline SCAS & $.58^{*}$ & .32 & $.54^{* * *}+$ & .37 & $.61^{* * *}+$ & $.58^{* * *}+$ \\
\hline $\mathrm{CDI}^{\mathrm{a}}$ & .22 & .29 & .11 & $.46^{* * *}+$ & .22 & $.44^{* *}$ \\
\hline SDQ Con & .13 & .31 & .21 & .23 & .06 & .28 \\
\hline
\end{tabular}

Notes: ASQ: Ambiguous Scenarios Questionnaire; Dis: Anticipated Distress, Th: Threat Interpretation; SCAS: Spence Children's Anxiety Scale; CDI: Children's Depression Inventory; SDQ Con: Strengths and Difficulties Questionnaire Conduct Problems subscale; + significant at $p<.05$ after Bonferonni correction (as significance values below .001 are unspecified by statistical programmes, associations that were significant at $\mathrm{p}<.001$ were considered a close enough approximation to the criteria set); ${ }^{\mathrm{a}}$ outlier excluded. Time 1-Time 1, $n=65$; Time 1-Time 2, $n=54$; Time 1 -Time 3, $n=49$; Time 2-Time 2, $n=54$; Time 3 -Time $3, n=49$.

Table 2. Mean scores on measures of anxious cognitions and affect

\begin{tabular}{|c|c|c|c|c|c|c|c|}
\hline & \multicolumn{2}{|c|}{$\begin{array}{l}\text { Time } 1 \\
n=65\end{array}$} & \multicolumn{2}{|c|}{$\begin{array}{l}\text { Time } 2 \\
n=54\end{array}$} & \multicolumn{2}{|c|}{$\begin{array}{l}\text { Time } 3 \\
n=49\end{array}$} & \multirow[b]{2}{*}{$F(2,41)$} \\
\hline & Mean $(S D)$ & Range & Mean $(S D)$ & Range & Mean $(S D)$ & Range & \\
\hline $\begin{array}{l}\text { ASQ Anticipated } \\
\text { Distress }\end{array}$ & $51.00(19.85)$ & $19-95$ & $51.22(22.70)$ & $0-101$ & $47.63(26.26)$ & $0-96$ & .22 \\
\hline $\begin{array}{l}\text { ASQ Threat } \\
\text { Interpretation } \\
\text { (composite) }\end{array}$ & $8.79(4.72)$ & $1-19$ & $6.65(4.84)$ & 0-19 & $6.15(5.52)$ & $0-21$ & $8.01^{* * *}$ \\
\hline SCAS & $24.54(12.28)$ & $3-55$ & $23.70(10.55)$ & $4-45$ & $23.03(14.20)$ & $1-56$ & 1.53 \\
\hline CDI & $5.66(4.79)$ & $0-20$ & $6.08(5.62)$ & $0-23$ & $5.52(5.21)$ & $0-18$ & $16.05^{* * *}$ \\
\hline SDQ Con & $2.04(1.83)$ & $0-6$ & $1.72(1.47)$ & $0-5$ & $2.00(1.78)$ & $0-7$ & 1.36 \\
\hline
\end{tabular}

Notes: ASQ: Ambiguous Scenarios Questionnaire; SCAS: Spence Children's Anxiety Scale; CDI: Children's Depression Inventory; SDQ con: Strengths and Difficulties Questionnaire Conduct Problems subscale) ${ }^{* *} p<.01 ;{ }^{* * *} p<.001$. 
Table 3. Predicting change in anxiety symptoms from ASQ anticipated distress dependent variable: Time 2 anxiety symptoms ${ }^{\mathrm{a}}$

\begin{tabular}{lrrrl}
\hline Predictor & \multicolumn{1}{c}{$\mathrm{B}$} & $\mathrm{SE}(\mathrm{B})$ & $\beta$ & Increment in $\mathrm{R}^{2}$ \\
\hline Step one(control) & & & & .60 \\
Time 1 SCAS & .57 & .08 & $.67^{* * *}$ & \\
Gender & 3.01 & 1.82 & .15 & \\
White UK & -3.54 & 1.90 & -.17 & \\
Step two & & & & .04 \\
Time 1 Anticipated & .13 & .05 & $.24^{*}$ & \\
$\quad$ distress & & & & \\
\hline
\end{tabular}

Notes: ASQ: Ambiguous Scenarios Questionnaire; SCAS: Spence Children's Anxiety Scale; ${ }^{*} p<.05,{ }^{* * *} p<.001$; ${ }^{\text {a }}$ outlier excluded.

indicating a large effect size support stability of individual differences for measures of anxious cognition and affect (time $1-$ time 2: ASQ Distress: $r(54)=.65, p<.001$; ASQ Threat: $r(54)=.66, p<.001$; SCAS: $r(54)=.68, p<.001$; time $1-$ time 3 : ASQ Distress: $r(49)=$ $.57, p<.001$; ASQ Threat: $r(49)=.61, p<.001$; SCAS: $r(49)=.74, p<.001$; time $2-$ time 3: ASQ Distress: $r(43)=.69, p<.001$; ASQ Threat: $r(43)=.76, p<.001$; SCAS: $r(43)=.82, p<.001)$. In addition, as seen in Table 2 , the concurrent associations between anxiety symptoms and anticipated distress were large and stable across time, $r$ 's ranged from. 53 to.61. There was, however, a trend for the association between threat interpretation and anxiety symptoms to increase over time (time 1:.23, time $2: .46$, time $3: .58$ ). Although not significant (time 1 to time $2: z=.95, p=.17$ ), this pattern does suggest that threat bias may be less central to anxiety in younger children.

Longitudinal associations between cognitive style and in anxiety symptoms in children after exposure to a major normative stress

Where bivariate correlations indicated a significant longitudinal relationship between cognitions and anxiety symptoms (see Table 1), multiple regression analyses were conducted to examine whether cognition predicted change in anxiety (i.e. predicted time 2 affect after controlling for time 1 anxiety). Associations between cognitions prior to the transition and anxiety shortly after (time 2) were investigated. To assess whether effects were maintained over a longer period of time, associations between time 1 and time 3 were analyzed. There were no significant interactions between gender or ethnicity and cognitions for any regression analyses (although the study was not powered to detect interactions). Reverse relationships were then investigated, i.e. whether earlier anxiety predicted later cognitions, following the same structure as described above (time 1 to 2 ; time 1 to 3 ).

\section{Time 1 Anxious cognitions and subsequent affect}

There was only one significant bivariate correlation between cognition prior to the transition and affect soon after: time 1 ASQ Distress and time 2 anxiety symptoms, $r(54)=.54$, $p<.001$. Regression analyses indicated that time 1 Distress cognitions significantly predicted time 2 anxiety symptoms, after controlling for gender, ethnicity and time 1 anxiety symptoms, $R^{2}=.67, F(4,48)=24.03, p<.001 ; \beta=.24, p=.02$ (see Table 3). The association 
Table 4. Predicting change in ASQ threat interpretation from anxiety symptoms dependent variable: Time 3 ASQ threat

\begin{tabular}{lrrrl}
\hline Predictor & \multicolumn{1}{c}{$\mathrm{B}$} & $\mathrm{SE}(\mathrm{B})$ & \multicolumn{1}{c}{$\beta$} & Increment in $\mathrm{R}^{2}$ \\
\hline Step one(control) & & & & .50 \\
Time 1 Threat & .66 & .13 & $.57^{* * *}$ & \\
interpretation & 3.39 & 1.16 & $.31^{* *}$ & \\
Gender & -1.83 & 1.21 & -.17 & \\
White UK & & & & .08 \\
Step two & & & & \\
Time 1 SCAS & .14 & .05 & $.33^{* *}$ & \\
\hline
\end{tabular}

Notes: ASQ: Ambiguous Scenarios Questionnaire; SCAS: Spence Children's Anxiety Scale; ${ }^{* *} p<.01,{ }^{* * *} p<.001$.

between anxious cognitions and subsequent affect was only slightly weaker at the subsequent assessment, $r(49)=.58, p<.001$; (see Table 2 ). Although regression analysis did not support a significant persisting association between time 1 Distress and change in anxiety symptoms from time 1 to time $3, R^{2}=.58, F(4,44)=17.71, p<.001 ; \beta=.17, p=.17$, the magnitude of effect was comparable (i.e..13 to.11). There were no significant associations between ASQ Threat and later anxiety scores.

\section{Time 1 affect and subsequent anxious cognitions}

There were no significant associations between anxiety symptoms at time 1 and cognitions at time 2 (i.e. over the transition event). On the other hand, there were significant bivariate correlations between time 1 anxiety symptoms and time 3 Threat and Distress cognitions. Time 1 anxiety symptoms significantly predicted time 3 Threat cognitions, after controlling for time 1 Threat, ethnicity and gender, $R^{2}=.55, F(4,44)=15.39, p<.001 ; \beta=.33$, $p=.005$ (see Table 4). Time 1 anxiety symptoms did not, however, predict change in Distress cognitions to time $3, R^{2}=.39, F(4,44)=8.75, p<.001 ; \beta=.11, p=.44$.

In summary, evidence of bi-directional links between cognitive bias and anxious affect was observed, but differed for Anticipatory Distress - which predicted change in anxiety but was not predicted by anxiety - and Threat - which was predicted by anxiety but did not predict change in anxiety.

\section{Discussion}

We conducted a short-term longitudinal study of threat interpretation and anticipatory distress and psychopathology symptoms to address three unresolved issues in developmental research. The findings concerning specificity were reasonably clear across all three timepoints: there was a consistently significant association between threat interpretation and anticipatory distress and anxiety symptoms that exceeded that found for depression or conduct problems. Findings addressing the second question about stability were equally clear: there was substantial stability of individual differences over the 1-year period; threat interpretation but not anticipatory distress showed an age-based decline over this period covering the 
transition to secondary school. [Results also hinted that the link between threat interpretation and anxiety symptoms may become stronger over time.] Finally, results addressing the third question about change over time were less clear and robust: anticipatory distress did predict an increase over time in anxiety symptoms; anxiety symptoms predicted an increase in subsequent threat interpretation.

The high level of stability over time and consistent associations with anxiety symptoms for Anticipated Distress supports the notion that this is a characteristic "style" that can be reliably elicited from late primary school children. Threat interpretation was also a stable characteristic within individuals, although its meaning vis-à-vis anxiety is less evident. Despite the longer time period covered, test-retest correlations were somewhat higher for cognitive variables than those reported from a community sample of 9-13 year olds (Muris et al., 2004) (.44.63). To establish whether this is a reliable difference requires replication, but studies of depression and aggression in youth suggest differences in stability of beliefs at different ages (e.g. Lochman and Dodge, 1994) which may account for differences between these two studies; for example, the inclusion of slightly younger children in the Muris study may have reduced the overall test-retest correlations. Notably, as a group there was a reduction over time in the extent to which events were interpreted as threatening, with higher scores at the pre-transition assessment than the post-transition assessment. Whether this represents an effect of age, development or the forthcoming secondary school transition cannot be disentangled by the current study, nor can potential confounding effects of characteristics of the secondary school transition. For example, this finding, and the emergence of significant associations between threat interpretation and anxiety in the post-transition assessments (i.e. age 11/12 years), may be consistent with the suggestion that there is a general bias towards threat information in childhood which non-anxious children learn to inhibit during mid-late childhood but which remains amongst more anxious children (e.g. Kindt and van den Hout, 2001).

Longitudinal analyses showed that Anticipated Distress predicted change in anxiety symptoms over time. In other words, children who anticipated a higher level of distress in response to ambiguous scenarios reported a greater subsequent increase in their anxiety symptoms. This is consistent with findings from treatment studies that suggest that anxious self-statements (based on the Negative Affectivity Self Statement Questionnaire, which includes items relating to both threat and distress) mediate change in the treatment of anxiety (Kendall and Treadwell, 2007). Our findings, however, suggest that the specific construct of anticipated distress may be particularly pertinent to the course of child anxiety symptoms, as longitudinal investigations suggested that prior anxiety predicted change in threat interpretation, and not vice versa. In children at this age, the concern that "a bad thing may happen", may be less influential on the development of anxiety than the concern that "I won't be able to cope with it". If replicated, this pattern may have implications for assessment, treatment and theory. For example, the observation that different cognitive components show differential longitudinal links with anxiety may go some way to mapping the connections between cognitive risk and symptoms and understanding which features are precursors versus consequences of anxiety. It may be, for example, that anxiety is preceded by a general nonspecific wariness or inhibition, associated with a view that one will not be able to cope, that is then subsequently accounted for by constructed explanations of internal experiences (i.e. theories about bad things that could happen), which may only later serve to maintain anxiety (e.g. Mathews and Mackintosh, 2000). 
It is important to note that whilst longitudinal findings may be consistent with causal relationships, alternative explanations for longitudinal associations exist (for example, associations with a third factor that explains both variables). Future research should employ both experimental studies and analyses of clinical change. Two studies to date have examined the change in anxious cognitions following cognitive-behavioural treatment for child anxiety with somewhat different results (Creswell, Schniering and Rapee, 2005; Waters, Wharton, Zimmer-Gembeck and Craske, 2008). Whilst both studies found reductions in "anxious cognitions" from pre-to post treatment assessments, Creswell et al. found a reduction in threat interpretation (amongst 7-15 year olds) which was not replicated by Waters et al. (812 years). They did not find a reduction in threat interpretation, but did find an increase in children's beliefs about their ability to influence events. Furthermore, Waters et al. reported a moderate association between change in influence cognitions and change in anxiety. The fact that a change in threat cognitions was only found in the study including adolescent children is intriguing in the light of the above discussion and highlights the importance of evaluating age effects in future research.

These findings and their implications need to be considered in the light of limitations of the study. The first limitation concerns generalizability. All participating children were recruited from schools in a single inner-London borough that represents a diverse population; this was not a clinic sample, and few children would be considered to show clinically significant anxiety. The possibility of non-linear effects and, specifically, whether the findings extend to clinical populations, requires further exploration. Whilst the rate of participation was low, importantly participants did not differ from non-participants on those demographic characteristics that were associated with anxiety symptoms (gender and ethnicity). We also focused specifically on interpretation of ambiguity; further exploration of other aspects of information processing known to be associated with anxiety is warranted (e.g. Hadwin et al., 2006). The sample sizes in the current study were too small to permit more complex longitudinal analyses such as structural equation modelling and latent growth curve models; nor did we have adequate power to detect interactions. Additionally, the final sample size would not allow for the detection of small-medium effects within the regression analyses employed and, as such, there is a possibility of Type II error and findings should therefore be regarded as preliminary. Like Muris et al. (2004) findings may also have been limited by the absence of a clear change in anxiety symptoms over the assessment period. Whilst we studied the children over a longer time period and included a significant life-event (the transition to secondary school), the means analysis cast doubt on the transition to secondary school as a general stress. The suggestion that the link between cognitive bias and anxious affect may be accentuated during periods of transition or vulnerability therefore requires further investigation, particularly more systematic assessment of cognitive bias around other normative and non-normative stresses (e.g. parental divorce) at specific child ages. Finally, as is commonly the case in research into anxiety and interpretation biases, all measures were based on child self-report. Future studies would benefit from more objective assessments of anxiety and interpretation that may be less prone to single informant bias. Balancing these limitations were a number of strengths, including the three occasions of measurement, assessment of anxiety symptoms and cognitions using multiple measures, and the issue of standard cognitive assessments of cognitive bias in children.

The current study provides further support for the association between children's interpretation biases and self-reported anxiety and stability of cognitions in late childhood. 
In addition, the study adds new information about the predictive role of cognitions in relation to change in anxiety over a 1-year period. More broadly, the study adds preliminary developmental data to the research on how characteristic "thinking styles" may precede change or respond to change in anxiety symptoms over time.

\section{Acknowledgments}

This work was carried out when Dr Creswell was based at University College London, funded by a fellowship from Camden and Islington NHS Trust. We would like to thank Islington Local Education Authority, the participating schools, the children, their parents and their teachers for all their help, Norah Breslin, Loanda Cullen, David Younger, Vicki Preston-Ladd and Katie Law for their assistance and Chris Brewin for his support and advice. We would also like to thank the anonymous reviewers for their helpful comments.

\section{References}

Barrett, P. M., Dadds, M. R. and Rapee, R. M. (1996). Family treatment of childhood anxiety: a controlled trial. Journal of Consulting and Clinical Psychology, 64, 333-342.

Barrett, P. M., Rapee, R. M., Dadds, M. M. and Ryan, S. M. (1996). Family enhancement of cognitive style in anxious and aggressive children. Journal of Abnormal Child Psychology, 24, 187-203.

Beck, A. T., Emery, G. and Greenberg, R. L. (1985). Anxiety Disorders and Phobias: a cognitive perspective. New York: Basic Books.

Bögels, S. M. and Zigterman, D. (2000). Dysfunctional cognitions in children with social phobia, separation anxiety disorder and generalised anxiety disorder. Journal of Abnormal Child Psychology, $28,205-211$.

Chorpita, B. F., Albano, A. M. and Barlow, D. H. (1996). Cognitive processing in children: relationship to anxiety and family influences. Journal of Clinical Child Psychology, 25, $170-176$.

Chung, H., Elias, M. and Schneider, K. (1998). Patterns of individual adjustment changes during middle school transition. Journal of School Psychology, 36, 83-101.

Creswell, C., O'Connor, T. and Brewin, C. (2006). A longitudinal investigation of maternal and child "anxious cognitions". Cognitive Therapy and Research, 30, 135-147.

Creswell, C., Schniering, C. and Rapee, R. (2005). Threat interpretation in anxious children and their mothers: group and treatment effects. Behaviour Research and Therapy, 43, 1375-1381.

Eley, T. C., Gregory, A. M., Lau, J. Y. F., McGuffin, P., Napolitano, M., Rijsdijk, F. V. and Clark, D. M. (2008). In the face of uncertainty: a twin study of ambiguous information, anxiety and depression in children. Journal of Abnormal Child Psychology, 36, 55-65.

Goodman, R. (1997). The Strengths and Difficulties Questionnaire: a research note. Journal of Child Psychology and Psychiatry, 38, 581-586.

Goodman, R. (2001). Psychometric properties of the Strengths and Difficulties Questionnaire. Journal of the American Academy of Child and Adolescent Psychiatry, 40, 1337-1345.

Hadwin, J. A., Garner, M. and Perez-Olivas, G. (2006). The development of information processing biases in childhood anxiety: a review and exploration of origins in parenting. Clinical Psychology Review, 26, 876-894.

Huesmann, L. R. and Guerra, N. G. (1997). Children's normative beliefs about aggression and aggressive behaviour. Journal of Personality and Social Psychology, 72, 408-419. 
Kazdin, A. E. (1981). Assessment techniques for childhood depression: a critical appraisal. Journal of the American Academy of Child and Adolescent Psychiatry, 20, 358-375.

Kendall, P. C. (1994). Treating anxiety disorders in children: results of a randomised clinical trial. Journal of Consulting and Clinical Psychology, 50, 672-689.

Kendall, P. C. and Treadwell, K. R. H. (2007). The role of self-statements as a mediator in treatment for youth with anxiety disorders. Journal of Consulting and Clinical Psychology, 75, 380-389.

Kindt, M. and Van Den Hout, M. (2001). Selective attention and anxiety: a perspective on developmental issues and the causal status. Psychopathology and Behavioral Assessment, 23, 193202.

Kovacs, M. (1979). The Children's Depression Inventory. Pittsburgh, PA: University of Pittsburgh School of Medicine.

Lansford, J. E., Malone, P. S., Dodge, K. A., Crozier, J. C., Pettit, G. S. and Bates, J. E. (2006). A 12-year prospective study of patterns of social information processing problems and externalising behaviours. Journal of Abnormal Child Psychology, 34, 715-724.

Lochman, J. E. and Dodge, K. A. (1994). Social-cognitive processes of severely violent, moderately aggressive, and nonaggressive boys. Journal of Consulting and Clinical Psychology, 62, 366-374.

Mathews, A. and Mackintosh, B. (2000). Induced emotional interpretation bias and anxiety. Journal of Abnormal Psychology, 109, 602-615.

Muris, P., Jacques, P. and Mayer, B. (2004). The stability of threat perception abnormalities and anxiety disorder symptoms in non-clinical children. Child Psychiatry and Human Development, 34, 251-265.

Muris, P., Luermans, J., Merckelbach, H. and Mayer, B. (2000). "Danger is lurking everywhere": the relation between anxiety and threat perception abnormalities in normal children. Journal of Behavior Therapy and Experimental Psychiatry, 31, 123-136.

Muris, P., Merckelbach, H. and Damsma, E. (2000). Threat perception bias in nonreferred, socially anxious children. Journal of Clinical Child Psychology, 29, 348-359.

Muris, P., Rapee, R. M., Meesters, C., Shouten, E. and Geers, M. (2003). Threat perception abnormalities in children: the role of anxiety disorders symptoms, chronic anxiety, and state anxiety. Journal of Anxiety Disorders, 17, 271-287.

Nolen-Hoeksema, S., Girgus, J. S. and Seligman, M. E. (1986). Learned helplessness in children: a longitudinal study of depression, achievement, and explanatory style. Journal of Personality and Social Psychology, 51, 435-442.

Nolen-Hoeksema, S., Girgus, J. S. and Seligman, M. E. P. (1992). Predictors and consequences of childhood depressive symptoms: a 5-year longitudinal study. Journal of Abnormal Psychology, 101, 405-422.

Robinson, N. S., Garber, J. and Hilsman, R. (1995). Cognitions and stress: direct and moderating effects on depressive versus externalizing symptoms during the junior high school transition. Journal of Abnormal Psychology, 104, 453-463.

Saylor, C. F., Finch, A. J., Spirito, A. and Bennett, B. (1984). The Children's Depression Inventory: a systematic evaluation of psychometric properties. Journal of Consulting and Clinical Psychology, 52, 955-967.

Shrout, P. E. and Fleiss, J. L. (1979). Intraclass correlations: uses in assessing rater reliability. Psychological Bulletin, 8, 420-428.

Spence, S. H. (1997). Structure of anxiety symptoms among children: a confirmatory factor-analytic study. Journal of Abnormal Psychology, 106, 280-297.

Spence, S. H. (1998). A measure of anxiety symptoms among children. Behaviour, Research and Therapy, 36, 545-566.

Tabachnick, B. G. and Fidell, L. S. (1996). Using Multivariate Statistics (3rd ed.). New York: Harper Collins College Publishers. 
Taghavi, M. R., Neshat-Doost, H. T., Moradi, A. R., Yule, W. and Dalgleish, T. (1999). Biases in visual attention in children and adolescents with clinical anxiety and mixed anxiety-depression. Journal of Abnormal Child Psychology, 27, 215-223.

Turner, J. E. and Cole, D. A. (1994). Developmental differences in cognitive diathesis for child depression. Journal of Abnormal Child Psychology, 22, 15-32.

Waters, A. M., Wharton, T. A., Zimmer-Gembeck, M. and Craske, M. (2008). Threat-based cognitive biases in anxious children: comparison with non-anxious children before and after cognitive-behavioural treatment. Behaviour Research and Therapy, 46, 358-374. 